J. Austral. Math. Soc. 21 (Series A) (1976), 504-507.

\title{
LATTICE POINTS IN A CONVEX SET OF GIVEN WIDTH
}

\section{Dedicated to Professor George Szekeres on the occasion of his 65th birthday}

G. B. ELKINGTON and J. HAMMER

(Received 20 January 1973; revised 15 July 1975)

Communicated by Jennifer Seberry Wallis

\section{1}

Let $S$ be a closed bounded convex set in $d$-dimensional Euclidean space $E^{d}$. The width $w(S)$ of $S$ is the minimum distance between supporting hyperplanes of $S$, and $L(S)$ is the number of integral lattice points in the interior of $S$.

If $a$ is a positive real number, we define

$$
g(a, d)=\min \{L(S): w(S)>a\} .
$$

Recently Scott (1973) has proved that

$$
g\left(\frac{2+\sqrt{3}}{2}, 2\right)=1
$$

In Section 2 of this note we prove that

$$
g(a, 2) \geqq\left[\frac{2 a}{2+\sqrt{3}}\right]^{2},
$$

where $[q]$ denotes the integral part of $q$. We also show that

$$
g(a, 2) \leqq\left[\frac{a^{2}}{\sqrt{3}}\right] .
$$

Earlier Sallee (1969) obtained a sharper result than Scott's (1) for sets of zonstant width in $E^{2}$. A set $W \subset E^{d}$ is said to have constant width $a$ if the distance between any two parallel supporting hyperplanes of $W$ equals $a$. From Sallee's result we have

$$
g^{*}(1.546,2)=1,
$$


where

$$
g^{*}(a, d)=\min \{L(W): w(W)>a\} .
$$

We have the following estimates for $g^{*}$ :

$$
g^{*}(a, 2) \geqq\left[\frac{a}{1.546}\right]^{2}
$$

and

$\left(3^{*}\right)$.

$$
g^{*}(a, 2) \leqq \frac{a^{2}}{2}(\pi-\sqrt{3}) .
$$

In Section 3 we prove an analogue of Minkowski's classical result. Let $K \subset E^{d}$ be a convex body which is central symmetric about the origin 0 . We define as before

$$
g_{0}(a, d)=\min \{L(K): w(K)>a\}
$$

Then

$$
g_{0}(2, d)=2 d+1
$$

and

$\left(2_{0}\right)$

$$
g_{0}(a, d) \sim \frac{\pi^{d / 2} \cdot a^{d}}{2^{d} \cdot \Gamma\left(\frac{d+2}{2}\right)} \quad \text { as } a \rightarrow x
$$

We now prove (2). Let $S$ be a closed bounded convex set in $E^{2}$ with $w(S)>a$. Write $r=[2 a /(2+\sqrt{3})]$. If $r=0$, the result is clear. So suppose $r \geqq 1$ and consider the similarity transformation

$$
S \rightarrow S^{\prime}=\frac{1}{r} S=\left\{\frac{1}{r} Y: Y \in S\right\} .
$$

Obviously,

$$
w\left(S^{\prime}\right)=\frac{1}{r} w(S)>\frac{a}{r} .
$$

Now let $T=\left(t_{1}, t_{2}\right)$ be a lattice point with $0 \leqq t_{1}, t_{2} \leqq r-1$ and consider the translate $S^{\prime \prime}$ of $S^{\prime}$ given by

$$
S^{\prime \prime}=S^{\prime}-\frac{1}{r} T=\left\{X-\frac{1}{r} T: X \in S^{\prime}\right\} .
$$


Obviously,

$$
w\left(S^{\prime \prime}\right)=w\left(S^{\prime}\right)>\frac{a}{r} \geqq \frac{2+\sqrt{3}}{2} .
$$

By (1), $S^{\prime \prime}$ contains a lattice point $G$. Hence $S^{\prime}$ contains the point $G+(1 / r) T$, and so $S$ contains the point $P=r(G+(1 / r) T)=r G+T$. But $T=\left(t_{1}, t_{2}\right)$ might have been chosen in $r^{2}$ different ways, for we could have selected each of $t_{1}, t_{2}$ in $r$ different ways. Therefore we have $r^{2}$ distinct lattice points $P=\left(p_{1}, p_{2}\right)$ in $S$. These are distinct, since $p_{1} \equiv t_{i}(\bmod r)(i=1,2)$ and the $t_{i}$ are a complete set of residue $\bmod r$. Hence $L(S) \geqq r^{2}$, from which we have (2).

The proof of $\left(2^{*}\right)$ is analogous to that of $(2)$, using $\left(1^{*}\right)$ instead of (1).

To prove (3) we use the following

Lemma 1. Let $R \subset E^{2}$ be a closed bounded measurable region. Then the minimum number of lattice points in $R$ is always less than the measure of $R$.

For the proof, see Theorem 3 in Niven and Zuckerman (1967).

Now the area of an equilateral triangle of width $a$ is $\left(a^{2} / \sqrt{3}\right)$, from which (3) follows.

We remark that this bound is the best we can obtain by making use of the lemma, since it is well-known that of all convex sets of a given width, the equilaterial triangle has the smallest area.

Analogously, the area of the Reuleaux triangle of constant width $a$ is $\frac{1}{2} a^{2}(\pi-\sqrt{3})$, from which $\left(3^{*}\right)$ follows.

\section{3}

Both $\left(1_{0}\right)$ and $\left(2_{0}\right)$ are simple consequences of the following

LEMMA 2. A central symmetric convex body $K \subset E^{d}$ centered at the origin, and of width a, contains the $d$-dimensional ball $U$ centred at the origin, of radius $a / 2$.

Since $b d K$ is a closed set, there is a point $P$ of it at a minimum distance $m$ from 0 . Then any supporting plane of $K$ at $P$ is normal to $0 P$, since otherwise there is a point of $b d K$ nearer to 0 than $P$. By the central symmetry, $w(K) \leqq 2 m$ and so $m \geqq \frac{1}{2} a$. The stated result follows.

We now prove $\left(1_{0}\right)$. Suppose that $K$ is a central symmetric convex body centred at 0 , of width exceeding 2 . By the lemma above, $K$ contains a $d$-dimensional ball $U$ of radius exceeding 1 , centred at 0 and hence $K$ contains, besides the origin, each of the $2 d$ points $(0, \cdots, 0, \pm 1,0, \cdots, 0)$. Hence $g(2, d) \geqq$ $2 d+1$. On the other hand, a $d$-dimensional ball of radius $s \quad(1<s<\sqrt{2})$ 
contains, besides the origin, precisely the $2 d$ points $(0, \cdots, 0, \pm 1,0, \cdots, 0)$, whence $g(2, d) \leqq 2 d+1$. This proves $\left(1_{0}\right)$.

It is well-known that the number of lattice points inside a $d$-dimensional ball $U$ of radius $r$ is asymptotically equal to its volume $\left.\pi^{d / 2} r^{d} / \Gamma((d+2) / 2)\right)$ as $r \rightarrow x$. An analogous argument to that just given, proves $\left(2_{0}\right)$.

\section{References}

I. Niven and H. S. Zuckerman (1967), 'Lattice points in regions', Proc. Amer. Math. Soc. 18, 364-370. G. T. Sallee (1969), 'The maximal set of constant width in a lattice', Pacific. J. Math. 28, 669-674.

P. R. Scott (1973), 'A lattice problem in the plane', Mathematika 20, 247-252.

Department of Pure Mathematics

University of Sydney

N.S.W., Australia 